\title{
Comparison of pain models to detect opioid-induced hyperalgesia
}

This article was published in the following Dove Press journal:

Journal of Pain Research

26 April 2012

Number of times this article has been viewed

\author{
Sumithra Krishnan' \\ Amy Salter ${ }^{2}$ \\ Thomas Sullivan ${ }^{2}$ \\ Melanie Gentgall ${ }^{3}$ \\ Jason White ${ }^{4}$ \\ Paul Rolan' \\ 'Discipline of Pharmacology, School \\ of Medical Sciences, The University \\ of Adelaide, ${ }^{2}$ Discipline of Public \\ Health, The University of Adelaide, \\ ${ }^{3}$ Pain and Anesthesia Research Clinic, \\ Royal Adelaide Hospital, ${ }^{4}$ Pharmacy \\ School, University of South Australia, \\ Adelaide, South Australia, Australia
}

Correspondence: Paul Rolan Discipline of Pharmacology, School of Medical Sciences, University of Adelaide, South Australia, 5005, Australia

Tel +61883134102

Fax +6I 882240685

Email paul.rolan@adelaide.edu.au
Objective: Chronic opioid therapy may be associated with hyperalgesia. Our objective was to determine if opioid-induced hyperalgesia detection sensitivity is dependent on the stimulus used to detect it.

Methods: This open design study compared the detection of hyperalgesia in opioid-dependent subjects $(n=16)$ and healthy control subjects $(n=16)$ using the following pain stimuli: cold pain, electrical stimulation, mechanical pressure, and ischemic pain. The opioid-dependent subjects were maintained on either methadone $(n=8)$ or buprenorphine $(n=8)$ for at least 3 months. None of the controls was dependent on opioids or other drugs of abuse.

Results: The opioid-dependent subjects were markedly more sensitive than controls to the cold pain test. Compared with the control group, the hazard ratio for ceasing the test due to intolerable pain was 7.7 (95\% confidence interval [CI] 2.6-23.3) in the buprenorphine group and 4.5 (95\% CI 1.7-15.6) in the methadone group, with similar data for the cold pain threshold. Of the remaining tests, there were differences only for the electrical pain threshold between treatment groups, with the geometric mean threshold in the buprenorphine group being 1.5 (95\% CI 1.1-1.9)-fold higher (ie, less sensitive) than that of the controls; the geometric mean for the methadone group was 1.3 (95\% CI 1.04-1.7)-fold higher than that of the controls. There were no significant differences between buprenorphine and methadone patients in test responses. Women were more sensitive to the cold pain (hazard ratio for tolerance, 3.1 [95\% CI 1.4-7.3]) and ischemic tests (hazard ratio for tolerance, 2.7 [95\% CI 1.2-6.1]). There were significant correlations between cold and ischemic tolerances $(\mathrm{r}=0.50 ; P=0.003)$ and between electrical and mechanical pain tolerances $(\mathrm{r}=0.52 ; P=0.002)$.

Conclusion: These findings indicate that cold pain is the most suitable of the methods tested to detect opioid-induced hyperalgesia. This is consistent with its sensitivity to detect opioid analgesia.

Keywords: opioid-induced hyperalgesia, opioid-dependent subjects, pain models

\section{Introduction}

Continued reporting of pain despite escalating doses of opioids has been interpreted as being due to pharmacological tolerance. However, recent evidence and studies on former opioid addicts, patients undergoing surgery, and healthy subjects suggest that exposure to opioids results in an increased sensitivity to pain, a phenomenon referred to as opioid-induced hyperalgesia $(\mathrm{OIH}) .{ }^{1}$ This offers an alternative explanation for why patients chronically administered opioids require increasing doses to attain adequate levels of analgesia. ${ }^{2}$ There are no proven management strategies to help these patients, apart from opioid dose reduction, with which many are unable to comply. ${ }^{3,4}$ 
To facilitate treatments for $\mathrm{OIH}$, accurate detection and measurement of its presence are needed. Human experimental pain models can enable measurement of the characteristics of $\mathrm{OIH}$ using standard protocols. With this approach, an investigator can control the experimentally induced pain (nature, localization, intensity, frequency, and duration of the stimulus) and provide quantitative measures of psychophysical, behavioral, and/or neurophysiological responses.

Previous studies have shown that $\mathrm{OIH}$ is dependent on the nature of the pain model used. Chu et $\mathrm{al}^{2}$ assessed the development of tolerance and OIH in six patients with chronic lower back pain. Patients were assessed before and 1 month after the commencement of oral morphine therapy. Cold pain and heat pain tests were used to measure pain sensitivity before and during a controlled infusion with remifentanil, a short-acting $\mu$ opioid agonist. All patients demonstrated an increase in sensitivity to experimental pain in relation to the cold pain test, showing reduced thresholds and tolerance, whereas OIH was not evident in the heat pain test.

Differences in the detection of OIH in relation to different pain induction methods were shown by Doverty et al. ${ }^{5}$ The nociceptive responses of methadone-maintained patients were compared to healthy control subjects. Electrical stimulation and cold pain tests were used as nociceptive stimuli. In the electrical stimulation test, methadone patients generally had a lower pain tolerance than controls at pre-dose, but had a higher tolerance 3-hours post-dose. With regard to the cold pain test, methadone patients were significantly less pain tolerant at both pre-dose and 3-hours post-dose.

Schall et $\mathrm{al}^{6}$ compared methadone patients with healthy controls using mechanical pressure induced pain. There was no substantial difference in pain threshold and tolerance between the groups; thus, OIH was not detected.

These results suggest that $\mathrm{OIH}$ is not consistently detected by all pain models, with the most sensitive and reliable test being cold pain. This is not necessarily a surprise. Using experimental pain models, detection of antinociception following opioid administration depends on the stimulus used. ${ }^{7}$ Comparisons across studies may be misleading due to differences in patient populations, investigator techniques, and sample size. It is also uncommon for studies of OIH to use more than one or two techniques. However, in this study, four experimental pain models are compared in terms of their ability to detect and measure $\mathrm{OIH}$.

\section{Materials and methods}

\section{Participants}

Sixteen opioid-dependent patients (ODS) (mean age, 37.4 years; range, 25-51 years) were enrolled. These included eight (four male and four female) methadone-maintained (mean dose, $85.25 \mathrm{mg}$; range, 35-150 mg) and eight (four male and four female) buprenorphine-maintained subjects (mean dose, $14.25 \mathrm{mg}$; range, 6-24 mg) who had been taking opioids for at least 3 months as part of an opioid substitution therapy program. Patients were recruited through an outpatient clinic from Drug and Alcohol Services South Australia, Parkside, South Australia, Australia. ODS were excluded if they were also dependent on alcohol or other drugs of abuse, had conditions that were affected by sensitivity to cold, had taken any analgesics (except their maintenance opioid) 24 hours prior to the study, or if they were suffering from chronic pain. A urine dipstick test (Microgenics, Passau, Germany) was performed on each subject to test for the presence of opioids (other than methadone or buprenorphine) and other drugs of abuse (cannabinoids, benzodiazepines, cocaine, and amphetamines).

Sixteen gender-matched healthy control subjects (eight male and eight female; mean age, 22.9 years; range, 18-37 years) were also recruited for the study from a healthy volunteer database. Control subjects were included if they were in good physical health and had no history of addiction or chronic opioid use. Potential control subjects were excluded if they suffered from chronic pain, had taken any analgesics 24 hours prior to the study, or if they were dependent on alcohol or other drugs of abuse. They also had a urine dipstick test to check for the presence of opioids and other drugs of abuse.

Before admission into the trial, all subjects gave informed consent. Subjects were reimbursed for their participation upon completion of the study and were free to withdraw at any time. The study was carried out in accordance with the principles of the International Conference of Harmonisation Good Clinical Practice, and was approved by the Royal Adelaide Hospital Research Ethics Committee, Adelaide, South Australia, Australia.

\section{Study plan and design}

Each subject was studied in one session, with each study session lasting for approximately two hours. All subjects had a brief familiarization session to accustom them to the experimental procedures of the pain models prior to testing. 
Pupillometry was performed before the commencement of testing. The tests were the cold pain test, electrical stimulation test, mechanical pressure test, and ischemic pain test. The pain tolerance and pain threshold for each test were measured. The order of the four pain tests was varied according to a balanced Latin square design to control for carry-over effects. The subject's eyes were covered during all tests, and there was a 15 -minute resting period between each pain test.

Upon completion of the testing schedule, the ODS took their usual dose of methadone or buprenorphine.

\section{Cold pain test}

The cold pain test was based on the procedures of Doverty et al. ${ }^{5}$ The test used two cylindrical temperature-controlled water baths $(380 \mathrm{~cm}$ in depth, $300 \mathrm{~cm}$ in diameter) of $34.5^{\circ} \mathrm{C}-35.5^{\circ} \mathrm{C}$ and $0.5^{\circ} \mathrm{C}-1.5^{\circ} \mathrm{C}$. A water pump (Brolga MV 1500, Brolga Australia Pty Ltd, Haberfield, NSW, Australia) was placed in the cold water bath to prevent laminar warming around the subject's immersed limb. Each subject's nondominant forearm and hand (fingers wide apart) were placed vertically into the warm water bath, controlled by a thermoregulator (Unistat 110, Thermoline Scientific, Sydney, Australia) for exactly 2 minutes. After 1 minute and 45 seconds, a blood pressure cuff was inflated to a pressure of $20 \mathrm{mmHg}$ below their diastolic blood pressure. This was done to minimize the role of blood flow in determining the reaction to cold. At exactly 2 minutes, their forearm was placed into the cold water bath. To minimize distraction and time cues, the subject's eyes were blindfolded for the entire procedure. Once the arm was immersed in the cold water bath, subjects indicated when they first experienced pain (pain threshold). They were asked to leave their arm submerged until they could no longer tolerate the pain (pain tolerance); the cut-off time limit was 180 seconds. Endpoints were measured as time (seconds).

\section{Electrical stimulation test}

The electrical stimulation test was based on the methods used by Doverty et al. ${ }^{5}$ A small amount of electrical contact gel (Livingstone International, Pty Ltd, Sydney, Australia) was applied to the subject's earlobe to provide conductance between the skin and electrode. The skin electrode, which delivered the electrical pulses, was clipped to the subject's ear. The subjects were blindfolded to minimize distraction. An electrical stimulator (Grass model SC6, Grass Instruments, Quincy, MA) delivered electrical square wave pulses of 14 milliseconds duration ( 0.7 pulses/second). The voltage commenced at 0 (baseline) and increased at a constant rate of 2 volts every 1.4 seconds (to a maximum of 100 volts). Subjects were asked to indicate when they first felt a sensation, when they first detected pain, and when they could no longer tolerate the pain. Endpoints were measured in volts.

\section{Mechanical pressure test}

A pressure algometer (Force One FDI, Wagner, Greenwich, CT) was used to measure mechanical pain. ${ }^{8-11}$ The algometer was pressed perpendicular to the skin above the thenar eminence (muscle on the palm of the hand, just beneath the thumb) on the nondominant hand of the subject. The pressure algometer was fixed on custom-made equipment adapted from a drill press, which allowed controlled and progressive force to be applied by the operator without fatigue. The force was increased at a fixed rate of 10 Newtons/10 seconds (to a maximum of 300 Newtons). Subjects indicated when they first felt pain and when they could no longer tolerate the painful stimulation. Endpoints were measured in Newtons.

\section{Ischemic pain test}

The ischemic pain test was based on the methods by Plesan et al. ${ }^{12} \mathrm{~A}$ blood pressure cuff was placed around the nondominant arm of the subject. The cuff pressure was increased to $20 \mathrm{mmHg}$ above the subject's systolic pressure. With the pressure maintained, subjects performed a handgrip exercise on an elastic ball, in accordance to the rhythm of a metronome (Wittner GmbH and Co, Isny im Allgäu, Germany). The subject's eyes were covered for the entire procedure to minimize distraction and time cues. Subjects were then asked to indicate when they first detected the pain and when they could no longer tolerate the pain (to a maximum of 5 minutes). Once pain tolerance was reached, the blood pressure cuff was immediately deflated. Endpoints were measured in seconds.

\section{Statistical analysis}

Statistical analysis was performed using SAS (v 9.2; SAS Institute Inc, Cary, NC) and statistical significance was assumed at $P<0.05$.

Differences in pain threshold and pain tolerance between drug groups and gender were assessed by Cox proportional hazards models for cold pain and ischemic pain and by twoway analysis of variance models for mechanical pressure pain and electrical stimulation pain. Data analyzed by analysis of variance were $\log$ transformed prior to model fitting to satisfy model assumptions. 
Associations between measures of pain (threshold and tolerance) for each pain stimulus were assessed by Spearman's correlation coefficients.

\section{Results}

\section{Pain threshold and tolerance}

Figure 1 shows the pain threshold and pain tolerance of the ODS and control subjects across the four pain models.

\section{Cold pain test}

There were significant treatment group differences in cold pain threshold and tolerance, with the opioid groups being more sensitive than the controls. For threshold, the hazard ratio for reporting pain was 6.8 (95\% CI 2.2-20.6) in the buprenorphine group compared with control; for the methadone group, the hazard ratio compared with control was 4.1 (95\% CI 1.4-11.7). For tolerance, the hazard ratio for terminating the test due to intolerable pain was 7.7 (95\% CI 2.6-23.3) in the buprenorphine group compared with control; for the methadone group, the hazard ratio compared with control was 4.5 (95\% CI 1.7-15.6).

\section{Electrical pain test}

There were significant treatment group differences in electrical threshold but not tolerance, with higher thresholds in the opioid groups. For threshold, the geometric mean in the buprenorphine group was 1.5 (1.1-1.9)-fold that of control, and for the methadone group, the geometric mean was 1.3 (95\% CI 1.04-1.7)-fold that of control.

\section{Ischemic pain test and mechanical pain}

There were no significant differences between treatment groups for either threshold or tolerance for ischemic and mechanical pain tests.

\section{Correlation among pain tests}

Correlations between thresholds and tolerances for each test are listed in Table 1. For each test, there was a moderate to high correlation between threshold and tolerance, as would be expected. There was a moderate correlation between cold pain and ischemic tolerances $(\mathrm{r}=0.50, P=0.003)$, and between electrical and mechanical tolerances $(\mathrm{r}=0.52$, $P=0.002)$ but other correlations were much weaker.

\section{Gender differences}

There was a significant gender difference apparent in the cold pain test, with the hazard ratio in women being 2.3 (1.0-5.4) for threshold and $3.1(1.4-7.3)$ for tolerance compared with men. For the ischemic test, the hazard ratio in women for threshold was 3.8 (1.5-9.6) and for tolerance was 2.7 (1.2-6.1) compared with men. For the mechanical pain test, there was no gender difference for threshold, but the tolerance was 1.4 (95\% CI 1.05-1.83)-fold higher for women compared with men. There was no significant difference between genders for either threshold or tolerance in the electrical pain test.

\section{Methadone versus buprenorphine subjects}

There were no appreciable differences in responses between the buprenorphine and methadone groups.

\section{Discussion}

Our results demonstrated that the nature of the pain stimulus is an important factor in detecting OIH across the pain modalities. Recently, Staahl and Drewes ${ }^{13}$ reviewed the literature on the use of experimental pain models to detect the effects of opioids in acute pain models and models of hyperalgesia (but not $\mathrm{OIH}$ ). The relative sensitivity of each model is not easy to determine, as it requires comparison between studies using different sample sizes and techniques; however, the vast majority of publications used only one pain model and very few compared several. OIH has not been studied experimentally in detail and our findings indicate that cold pain is the most sensitive of the methods tested in detecting opioid-related hyperalgesia. This was evident by the marked increase in sensitivity to cold pain threshold and tolerance by the ODS compared with the controls. The only other difference detected was reduced sensitivity to electrical pain threshold but not tolerance.

Our findings are consistent with results from previous studies, demonstrating that different pain stimuli produce different magnitude and direction of changes in pain tolerance between healthy participants and opioid users. A study conducted by Doverty et al ${ }^{5}$ compared nociceptive responses between 16 methadone-maintained and 16 healthy control subjects using the electrical stimulation and cold pain models. Their findings support our results because the methadonemaintained subjects had a lower tolerance to cold pain and a higher tolerance to electrical pain in comparison to controls; hence, demonstration of OIH is dependent on the test model. Compton et a $1^{14}$ compared the pain tolerance of 18 methadone, 18 buprenorphine, and 18 control subjects using the cold pain test. The opioid-maintained subjects were significantly less tolerant to cold pain than controls, which is also consistent with our findings. However, Pud et a $\mathrm{l}^{15}$ showed 
A

Cold pain threshold

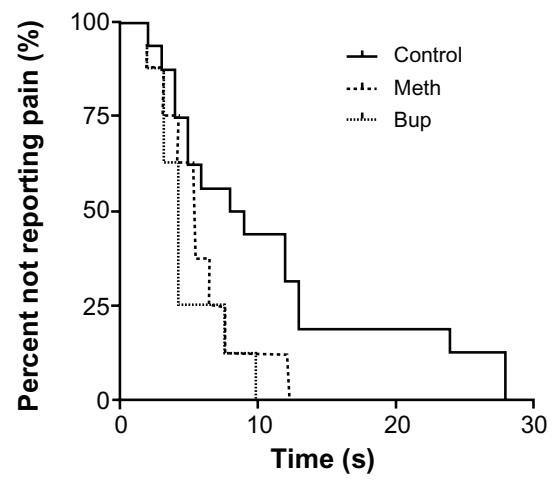

C

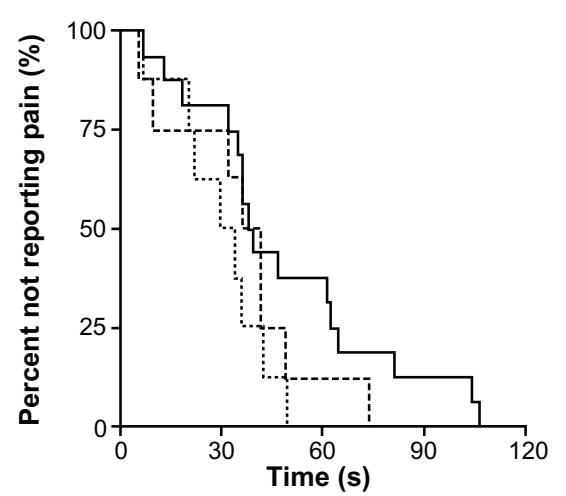

E

Electrical stimulation
threshold and tolerance

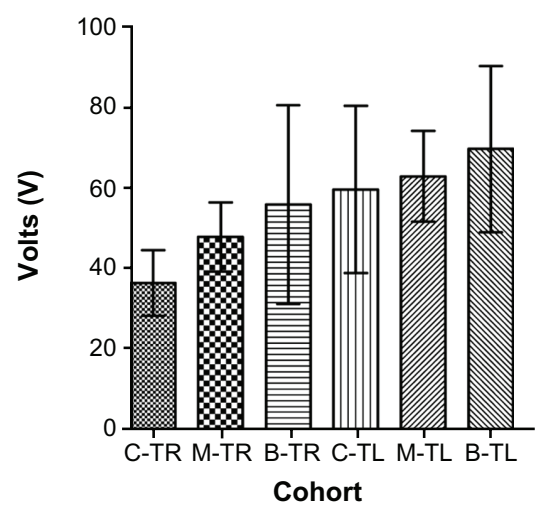

B

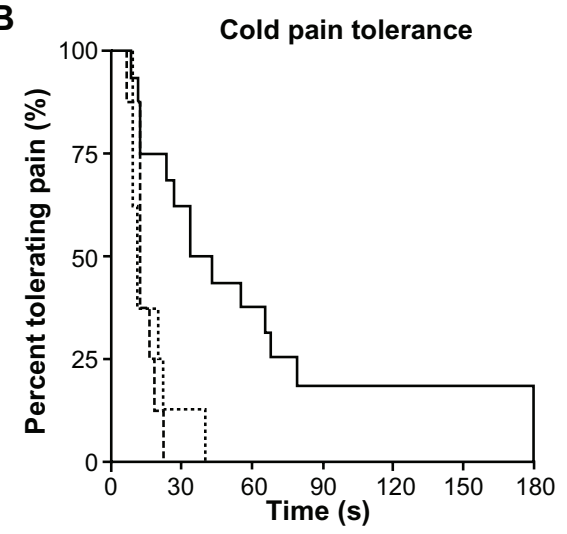

D

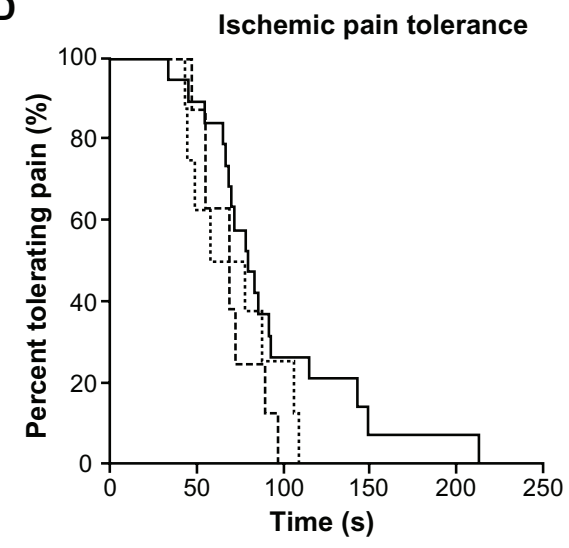

$\mathbf{F}$

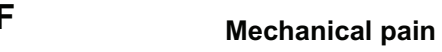
threshold and tolerance

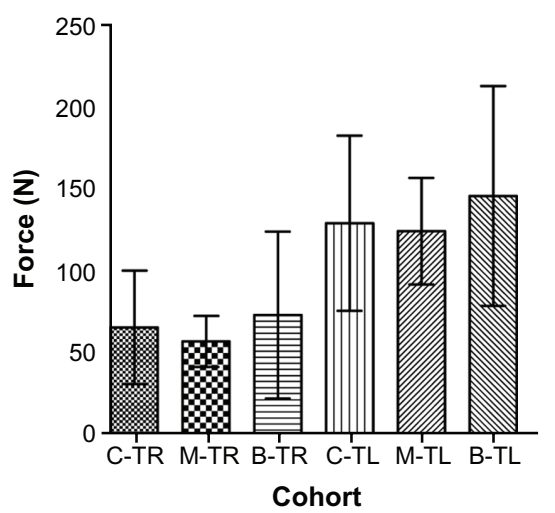

Figure I Comparison of pain threshold and pain tolerance between control subjects $(n=16)$, methadone dependent subjects $(n=8)$, and buprenorphine dependent subjects $(n=8)$ among the four different pain modalities. (A-D) depict cold pain threshold, cold pain tolerance, ischemic pain threshold, and ischemic pain tolerance, respectively, using Kaplan-Meier survival curves. (E and $\mathbf{F}$ ) represent the electrical stimulation threshold and tolerance and the mechanical pain threshold and tolerance, presented as the mean \pm standard deviation. Abbreviations: Meth, methadone-dependent subjects; Bup, buprenorphine-dependent subjects; C-TR, controls' threshold; M-TR, methadone-dependent subjects' threshold; B-TR, buprenorphine-dependent subjects' threshold; C-TL, controls tolerance; M-TL, methadone-dependent subjects' tolerance; B-TL, buprenorphine-dependent subjects' tolerance.

that although opioid addicts had a shorter cold pain tolerance time than control subjects, consistent with our findings, they had a significantly higher threshold time, which is not consistent with our findings. A possible explanation for their higher threshold time could be that addicts may first try to deny the feelings of pain (high threshold), but once this is no longer possible, they react quickly, as confirmed by their low pain tolerance time.

Luginbuhl et $\mathrm{al}^{7}$ used five pain tests (electrical pain, cold pain, pressure pain, ischemic pain, and heat pain) to measure 
Table I Correlations between models

\begin{tabular}{|c|c|c|c|c|c|c|c|c|}
\hline & $\begin{array}{l}\text { Cold } \\
\text { threshold }\end{array}$ & $\begin{array}{l}\text { Cold } \\
\text { tolerance }\end{array}$ & $\begin{array}{l}\text { Ischemic } \\
\text { threshold }\end{array}$ & $\begin{array}{l}\text { Ischemic } \\
\text { tolerance }\end{array}$ & $\begin{array}{l}\text { Mechanical } \\
\text { threshold }\end{array}$ & $\begin{array}{l}\text { Mechanical } \\
\text { tolerance }\end{array}$ & $\begin{array}{l}\text { Electrical } \\
\text { threshold }\end{array}$ & $\begin{array}{l}\text { Electrical } \\
\text { tolerance }\end{array}$ \\
\hline \multicolumn{9}{|c|}{ Spearman correlation coefficients $(n=32)$} \\
\hline \multirow[t]{2}{*}{ Cold threshold } & 1.00 & 0.58 & 0.11 & 0.09 & 0.37 & 0.22 & 0.07 & -0.10 \\
\hline & & 0.0005 & 0.53 & 0.62 & 0.04 & 0.22 & 0.72 & 0.60 \\
\hline \multirow[t]{2}{*}{ Cold tolerance } & 0.58 & 1.00 & 0.46 & 0.50 & 0.31 & 0.38 & -0.25 & 0.18 \\
\hline & 0.0005 & & 0.009 & 0.003 & 0.09 & 0.03 & 0.16 & 0.33 \\
\hline \multirow[t]{2}{*}{ Ischemic threshold } & 0.11 & 0.46 & 1.00 & 0.57 & -0.02 & 0.21 & -0.25 & 0.16 \\
\hline & 0.53 & 0.009 & & 0.0006 & 0.93 & 0.25 & 0.16 & 0.39 \\
\hline \multirow[t]{2}{*}{ Ischemic tolerance } & 0.09 & 0.50 & 0.57 & 1.00 & 0.19 & 0.34 & -0.22 & 0.19 \\
\hline & 0.62 & 0.003 & 0.0006 & & 0.30 & 0.05 & 0.22 & 0.30 \\
\hline \multirow[t]{2}{*}{ Mechanical threshold } & 0.37 & 0.31 & -0.016 & 0.19 & 1.00 & 0.80 & 0.28 & 0.43 \\
\hline & 0.038 & 0.09 & 0.93 & 0.30 & & $<0.0001$ & 0.11 & 0.01 \\
\hline \multirow[t]{2}{*}{ Mechanical tolerance } & 0.23 & 0.38 & 0.21 & 0.34 & 0.80 & 1.00 & 0.21 & 0.52 \\
\hline & 0.22 & 0.03 & 0.25 & 0.05 & $<0.0001$ & & 0.25 & 0.002 \\
\hline \multirow[t]{2}{*}{ Electrical threshold } & 0.066 & -0.25 & -0.25 & -0.22 & 0.28 & 0.21 & 1.00 & 0.56 \\
\hline & 0.72 & 0.16 & 0.16 & 0.22 & 0.11 & 0.25 & & 0.0009 \\
\hline \multirow[t]{2}{*}{ Electrical tolerance } & -0.098 & 0.18 & 0.16 & 0.19 & 0.43 & 0.52 & 0.56 & 1.00 \\
\hline & 0.60 & 0.33 & 0.39 & 0.30 & 0.01 & 0.002 & 0.0009 & \\
\hline
\end{tabular}

Note: In each cell, the upper figure is the Spearman rho and the lower figure the $P$ value.

the analgesic effects of alfentanil in 14 healthy volunteers (seven placebo and seven receiving alfentanil). There was no significant drug effect on the ischemic pain and heat pain model, whereas a significant difference was detected between the electrical, cold, and pressure pain tests. Although this was using an acute pain test rather than one of $\mathrm{OIH}$, these findings confirm our finding that the opioid effects in one pain test cannot be extrapolated to another.

Reznikov and colleagues ${ }^{10}$ found no significant difference in pain thresholds between opioid maintained patients and patients receiving non-opioid analgesics using the mechanical pressure test, which is also consistent with our findings.

Reasons for the discrepancies among the different outcomes of the different pain models observed in the various studies can be attributed in part to the nature of the pain stimuli.

Cold pain is an example of a tonic pain model. The innocuous cool sensation and cold pain are mediated by two different types of primary afferent fibers: ${ }^{16} \mathrm{~A}-\delta$ fibers mediate cold sensations and C-fibers mediate cold pain. ${ }^{13}$ The combination of the A- $\delta$ and C-fiber activation results in aching pain.

As mentioned, the cold pain test appears to be highly sensitive in detecting opioid effects. However, it is not a universally sensitive pain model, as it does not detect the effects of the potent high-efficacy non-steroidal anti-inflammatory drug ketorolac. ${ }^{14}$

Although the cold pain model has good reliability and validity and effectively mimics chronic pain conditions due to its unpleasantness, small variations in water temperature (as little as $2^{\circ} \mathrm{C}$ ) can result in a significantly different pain experience. ${ }^{17}$ This methodological problem could explain some of the quantitative differences in the pain threshold and tolerance results, but should not affect the direction of the change.

Electrical stimulation is an example of a phasic pain model. An advantage of this stimulus is that it directly activates nerve fibers instead of specific nociceptor activation. ${ }^{13}$ Unlike the other three tests, which were applied to the upper limb, in this study, we used the earlobe site to be consistent with Doverty et al. ${ }^{5}$ Although this might affect the magnitude of the effect, we think that it is unlikely to be the reason for the ODS having higher threshold and tolerance than the controls, compared with the cold pain test in which the direction of difference was reduced. However, this is a potential factor in the observed higher tolerance in the ODS in this test compared with the others and future work should also include a similar test in the upper limb.

The mechanical pressure stimulus activates sensitive mechanoreceptors, myelinated A- $\delta$ fibers, and unmyelinated C-fibers, which mediate a dull, aching pain. It is experimentally equivalent to palpations in the clinical setting. ${ }^{18}$ A limitation of the mechanical pressure model is that the stimulus is not specific. Non-specific receptors in skin and deep tissue are activated along with non-nociceptors, which could have an inhibitory effect on pain perception. Additionally, when mechanical pressure is truly nociceptive, it is likely to produce tissue damage. ${ }^{13}$ Mechanical stimulation 
can also be tonic (squeezing) or phasic (impact). Therefore, the use of either tonic or phasic mechanical stimuli could lead to variations in results.

Ischemic pain is a form of tonic muscle pain. It is activated by a combination of $\mathrm{A}-\delta$ and $\mathrm{C}$ nociceptors from deep tissues. ${ }^{19} \mathrm{~A}$ limitation of this model is that although it induces pain in muscles, it is non-specific since it activates skin and other tissues, contributing to the pain experience. In addition to activating nociceptors, the contact of the tourniquet on the skin can also activate low threshold non-nociceptive nerves, which can exert an inhibitory effect on pain mechanisms. This model has not been used extensively with opioids. ${ }^{13}$

In addition to differing $\mathrm{OIH}$ detection sensitivities, the results from the four tests showed weak correlation, except for correlation between cold pain and ischemic tolerances and between electrical and mechanical tolerances. Other authors have also found relatively weak correlation between pain tests. For example, Fillinghim and colleagues ${ }^{20}$ found that analgesic responses in women using heat pain correlated with ischemic and pressure pain. However, Bhalang et $\mathrm{al}^{8}$ showed a weak correlation between ischemic pain and heat with pressure pain. Similarly, Hastie et $\mathrm{al}^{9}$ found that correlations across pain stimuli were weak when multiple tests were conducted in the same individual. These studies, along with our data, show that findings from one test cannot be extrapolated to another, and most importantly, that absence of an effect cannot be concluded from one pain model.

Women were more sensitive to cold pain and ischemic pain. Gender differences in experimental pain sensitivity

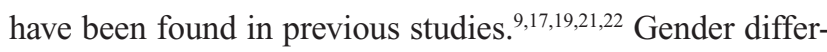
ences between pain tolerance may be due to women having higher levels of anxiety associated with experimental pain in comparison to clinical pain. ${ }^{20}$ The gender of the investigator inflicting the pain is an additional factor. ${ }^{17} \mathrm{~A}$ study by Gijsbers and Nicholson ${ }^{23}$ demonstrated that males had a higher pain threshold when tested by a female investigator than by a male investigator, whereas there was no difference in the pain thresholds of female subjects regardless of the gender of the investigator. This conclusion further supports earlier research conducted by Kallai et $\mathrm{al}^{24}$ and Levine and De Simone. ${ }^{25}$ In our study, we only had one female investigator, so subjectinvestigator gender differences could not be concluded.

We selected patients receiving opioid substitution therapy rather than pain patients receiving opioids for this study to avoid the potential confounding effects of the pain states on hyperalgesia. One additional factor to consider in the interpretation of this study is that the mean age of ODS participants was 14 years older than the controls. There is no suitable information in the literature on whether an age difference of this size affects the test results. However, the primary objective was to compare the ability of the tests to differentiate between the groups. The observation that one test did this well (cold pain) and the others did not supports the design and conclusions of the study.

Our results also showed that methadone- and buprenorphinemaintained subjects were equally hyperalgesic. This was supported by Compton et al, ${ }^{14}$ who found no significant difference in cold pain tolerance times between methadonemaintained and buprenorphine-maintained subjects.

As hypothesized, the sensitivity of pain models in detecting $\mathrm{OIH}$ varied between the different methods due to the nature of the nociceptive stimulus. These findings indicate that the cold pain test is the most effective pain model in detecting OIH and should be the preferred model in research studies investigating OIH. Furthermore, subject gender and/or subject-investigator gender match/mismatch must be considered as important factors in the design of future experimental pain studies.

\section{Acknowledgments}

We would like to acknowledge the assistance of Mr Aaron Farquharson, who was extremely helpful in assisting with the conducting of this study.

\section{Disclosure}

The authors reports no conflicts of interest in this work.

\section{References}

1. Angst MS, Clark J. Opioid-induced hyperalgesia: a qualitative systematic review. Anesthesiology. 2006;104:570-587.

2. Chu LF, Clark DJ, Angst MS. Opioid tolerance and hyperalgesia in chronic pain patients after one month of oral morphine therapy: a preliminary prospective study. J Pain. 2006;7:43-48.

3. Mercadante S, Bianchi M, Villari P. Opioid plasma concentration during switching from morphine to methadone preliminary data. Support Care Cancer. 2003;11:326-331.

4. Gardell LR, King T, Ossipov MH. Opioid receptor-mediated hyperalgesia and antinociceptive tolerance induced by sustained opiate delivery. Neurosci Lett. 2006;396:44-49.

5. Doverty M, White JM, Somogyi AA. Hyperalgesic responses in methadone maintenance patients. Pain. 2001;90:91-96.

6. Schall U, Katta T, Pries E. Pain perception of intravenous heroin users on maintenance therapy with levomethadone. Pharmacopsychiatry. 1996;29:176-179.

7. Luginbuhl M, Schnider TW, Petersen-Felix S. Comparison of five experimental pain tests to measure analgesic effects of alfentanil. Anesthesiology. 2001;95:22-29.

8. Bhalang K, Sigurdsson A, Slade GD. Associations among four modalities of experimental pain in women. J Pain. 2005;6:604-611.

9. Hastie BA, Riley JL, Robinson ME. Cluster analysis of multiple experimental pain modalities. Pain. 2005;116:227-237.

10. Reznikov I, Pud D, Eisenberg E. Oral opioids administration and hyperalgesia in patients with cancer or chronic non-malignant pain. Br J Clin Pharmacol. 2005;60:311-318. 
11. Rolke R, Magerl W, Campbell KA. Quantitative sensory testing: a comprehensive protocol for clinical trials. Eur J Pain. 2006;10:77-88.

12. Plesan A, Sollevi A, Segerdahl M. The N-methyl-D-aspartatereceptor antagonist dextromethorphan lacks analgesic effect in a human experimental ischemic pain model. Acta Anaesthesiol Scand. 2000;44:924-928.

13. Staahl C, Drewes AM. Experimental human pain models: a review of standardized methods for preclinical testing of analgesics. Basic Clin Pharmacol Toxicol. 2004;95:97-111.

14. Compton P, Charuvastra VC, Ling W. Pain intolerance in opioidsmaintained former opiate addicts: effect of long-acting maintenance agent. Drug Alcohol Depend. 2001;63:139-146.

15. Pud D, Cohen D, Lawental. Opioids and abnormal pain perception. New evidence from a study of chronic opioids addicts and healthy subjects. Drug Alcohol Depend. 2006;82:218-223.

16. Simone DA, Kajander KC. Responses of cutaneous A-fiber nociceptors to noxious cold. J Neurophysiol. 1997;77:2049-2060.

17. Mitchell LA, MacDonald RA, Brodie EE. Temperature and the cold pressor test. J Pain. 2004;5:233-237.

18. Ringler R, Greiner M, Kohlloeffel L. BOLD effects in different areas of the cerebral cortex during painful mechanical stimulation. Pain. 2003; 105:445-453.
19. Fillingim RB, Gear RW. Sex differences in opioids analgesia: clinical and experimental findings. Eur J Pain. 2004;8:413-425.

20. Fillinghim RB, Ness TJ, Glover TL. Experimental pain models reveal no sex differences in pentazocine analgesia in humans. Anesthesiology. 2004;100:1263-1270.

21. Rollman GB, Abdel-Shaheed J, Gillesphie JM. Does past pain influence current pain: biological and psychosocial models of sex differences. Eur J Pain. 2004;8:427-433.

22. Romberg R, Olofsen E, Sarton E. Pharmacokinetic-pharmacodynamic modeling of morphine-6-glucoronide-induced analgesia in healthy volunteers: absence of sex differences. Anesthesiology. 2004;100:120-133.

23. Gijsbers K, Nicholson F. Experimental pain thresholds influenced by sex of experimenter. Percept Mot Skills. 2005;101:803-807.

24. Kallai I, Barke A, Voss U. The effects of experimenter characteristics on pain reports in women and men. Pain. 2004;112:142-147.

25. Levine FM, De Simone LL. The effects of experimenter gender on pain report in male and female subjects. Pain. 1991;44:69-72.
Journal of Pain Research

\section{Publish your work in this journal}

The Journal of Pain Research is an international, peer-reviewed, open access, online journal that welcomes laboratory and clinical findings in the fields of pain research and the prevention and management of pain. Original research, reviews, symposium reports, hypothesis formation and commentaries are all considered for publication.

\section{Dovepress}

The manuscript management system is completely online and includes a very quick and fair peer-review system, which is all easy to use. Visit http://www.dovepress.com/testimonials.php to read real quotes from published authors. 